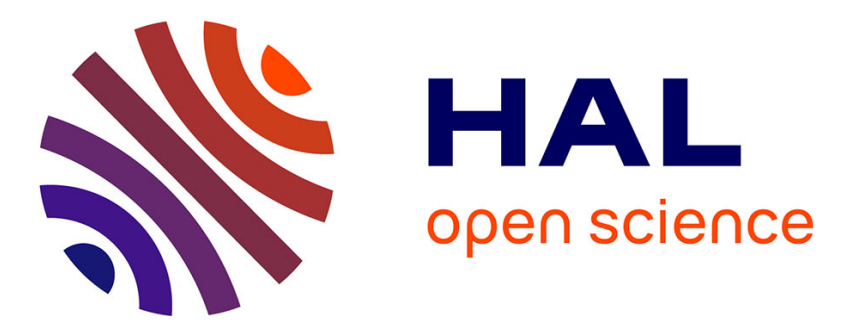

\title{
An algorithm for the computation of multiple Hopf bifurcation points based on Pade approximants
}

Grégory Girault, Yann Guevel, Jean-Marc Cadou

\section{To cite this version:}

Grégory Girault, Yann Guevel, Jean-Marc Cadou. An algorithm for the computation of multiple Hopf bifurcation points based on Pade approximants. International Journal for Numerical Methods in Fluids, 2012, 68 (9), pp.1189-1206. 10.1002/fld.2605 . hal-00715976

\section{HAL Id: hal-00715976 \\ https://hal.science/hal-00715976}

Submitted on 21 Jun 2018

HAL is a multi-disciplinary open access archive for the deposit and dissemination of scientific research documents, whether they are published or not. The documents may come from teaching and research institutions in France or abroad, or from public or private research centers.
L'archive ouverte pluridisciplinaire HAL, est destinée au dépôt et à la diffusion de documents scientifiques de niveau recherche, publiés ou non, émanant des établissements d'enseignement et de recherche français ou étrangers, des laboratoires publics ou privés. 


\title{
An algorithm for the computation of multiple Hopf bifurcation points based on Pade approximants
}

\author{
G. Girault ${ }^{1,2}$, Y.Guevel $^{2}$ and J. M. Cadou ${ }^{2}$ \\ ${ }^{1}$ Centre de recherche des Ecoles de Saint-Cyr Coëtquidan, Ecoles Militaires de Coëtquidan, Guer cedex, France \\ ${ }^{2}$ Laboratoire d'Ingénierie des Matériaux de Bretagne, Université Européenne de Bretagne, Université de Bretagne Sud, \\ Rue de Saint Maudé, Lorient Cedex, France
}

\begin{abstract}
Recently, a numerical method was proposed to compute a Hopf bifurcation point in fluid mechanics. This numerical method associates a bifurcation indicator and a Newton method. The former gives initial guesses to the iterative method. These initial values are the minima of the bifurcation indicator. However, sometimes, these minima do not lead to the convergence of the Newton method. Moreover, as only a single initial guess is obtained for each computation of the indicator, the computational time to obtain a Hopf bifurcation point can be quite long. The present algorithm is an enhancement of the previous one. It consists in automatically computing several initial guesses for each indicator curve. The majority of these initial values leads to the convergence of the Newton method. This method is evaluated through the problem of the lid-driven cavity with several aspect ratios in the framework of the finite element analysis of the 2D Navier-Stokes equations. The results prove the efficiency and the robustness of the proposed algorithm.
\end{abstract}

\section{INTRODUCTION}

This paper focuses on the numerical predictions of a Hopf bifurcation for incompressible viscous flows. Such instability occurs when a stationary flow becomes a time-periodic one.

From a mathematical point of view, if $\sigma$ stands for a complex eigenvalue of the Jacobian matrix, then a Hopf bifurcation occurs when the real part of two eigenvalues becomes null for a critical Reynolds number, noted $\mathrm{Re}_{\mathrm{c}}$.

The numerical methods used to compute accurately such instabilities are divided into two categories: the direct methods and the indirect ones with the monitoring of an indicator. The latter consists, for example, in computing the eigenvalues of Jacobian matrix and determining when a pair of complex conjugated values crosses the imaginary axis [4,5]. In [2], the authors have proposed a bifurcation indicator which has the property to be null at a Hopf bifurcation point. This method gives accurate values of a bifurcation point, but it requires much calculi and, consequently, much central processing unit (CPU) time. Let us remark that a stationary bifurcation indicator also exists and has been successfully used to study the Coanda effect [6].

In the direct methods [3,7], a Newton algorithm is used to iteratively determine the solution of a nonlinear problem which exactly satisfies a Hopf bifurcation point. The drawback of this approach is that the convergence of the iterative method is governed by the quality of the computed initial 
guesses. Recently, in [1], the authors proposed to link the indicator proposed in [2] and the Newton method. The principle of this method, which can be denoted as a hybrid method, is that the bifurcation indicator automatically provides an initial value to the iterative method. This initial guess is the minimum value of the indicator which is computed for a fixed value of the Reynolds number and only depends on the frequency. Although the majority of these initial values permits the computing of a Hopf bifurcation point, it does happen that, for some candidates, the Newton method fails to converge. As the bifurcation indicator only gives a single value for the Newton method per computation, a significant amount of computing time is then needed without assuring that this value is a good initial guess.

The purpose of the study is to develop an improved algorithm which allows one to compute Hopf bifurcation points in an automatic and robust way. As in [1], this algorithm combines both indirect and direct methods. Thus, the indirect method helps isolate several admissible initial values, and the direct method efficiently performs a Newton calculation. The initial values are determined as minima of an indicator curve, noted $\phi_{\operatorname{Re}}(\omega)$, after the computation of which the first and second derivatives respect the pulsation $\omega$ of the flow. These indicator curves are determined by using a perturbation method (the asymptotic numerical method [8]) associated to rational fractions called Padé approximants $[9,10]$. As these indicator curves are analytical functions of the angular frequency, their minima are easily determined by computing the roots of the numerator of the Padé approximants.

The proposed method permits one to give more than one initial value per indicator computation. This lets one increase the number of possible bifurcation points without increasing the computational times. Compared to the previous study [1] and other existing algorithms, the presented method is characterized by its robustness and reliability.

This paper is divided into three main parts. The first part is devoted to theoretical aspects for incompressible viscous flow and stability analysis. The second part of the paper recalls the fundamentals of asymptotical numerical methods. The proposed algorithm, based on the use of an indicator curve and its derivative functions, is exposed. Finally, some classical results for the academic problem of the lid-driven cavity are presented. They show the efficiency and the accuracy of the studied method.

\section{THEORY}

\subsection{Equations to be solved}

The movement of a laminar incompressible flow in two dimensions is described by the NavierStokes equations:

$$
\left\{\begin{array}{cc}
\dot{u}_{i}-v u_{i, j j}+u_{j} u_{i, j}+\frac{1}{\rho} p_{, i}=0 & \text { in } \Omega \\
u_{i, i}=0 & \text { in } \Omega \\
u=\lambda u_{d} & \text { on } \partial_{u} \Omega
\end{array}\right.
$$

In Equation (1), $u_{i}, p, \rho$ and $v$ stand, respectively, for the velocity in the ith-direction, the pressure, the fluid density and the kinematic viscosity. The notations $\left(\bullet^{\bullet}\right)$ and $(\bullet, i)$ stand for the derivatives with respect to time and space, respectively, in the direction $i$. The term $\lambda$ is a control parameter indicating the intensity of the imposed velocity and can be identified with the Reynolds number $\operatorname{Re}=u L / v$ using a reference length $L$.

Equation (1) can be written in the following form:

$$
M(\dot{U})+L(U)+Q(U, U)-\lambda F=0 \quad \text { in } \Omega
$$

where $U$ designates a concatenated vector containing the velocity $u$ and the pressure $p$, and $M$ is a mass matrix. $L(U)$ is a linear operator containing the pressure and diffusion terms, whereas the quadratic operator $Q(U, U)$ contains the convective terms of the Navier-Stokes equations. The term $\lambda F$ stands for a load vector proportional to the imposed velocity on $\partial_{u} \Omega$. The reader can find explicit descriptions of these operators in [2]. 
The stability of the flow is studied by adding a perturbation term $\mathrm{V}(t)$ to the stationary solution $U^{S}$ of the Navier-Stokes Equation (2). For a Hopf bifurcation, this perturbation term is written as follows:

$$
\vee(t)=V(\mathbf{x}) e^{i \omega t}
$$

In expression (3), $\omega$ and $V$ stand, respectively, for the pulsation and the complex mode of the perturbation. The vector $\mathbf{x}$ designates the spatial coordinates. Introducing term Equation (3) in the Navier-Stokes Equation (2) and neglecting quadratic and higher order terms in V, the following system is obtained:

$$
\left\{\begin{array}{cc}
i \omega M(V)+L(V)+Q\left(V, U^{S}\right)+Q\left(U^{S}, V\right)=0 & \text { in } \Omega \\
V_{U}=0 & \text { on } \partial_{U} \Omega \\
L\left(U^{S}\right)+Q\left(U^{S}, U^{S}\right)-\lambda^{S} F=0 & \text { in } \Omega
\end{array}\right.
$$

The solution of system (4) is the vector $\Lambda=\left\{V^{a}, V^{b}, \omega, U^{S}\right\}$, which corresponds to a Hopf bifurcation for the studied stationary flow. The notations $V^{a}$ and $V^{b}$ stand, respectively, for the real and imaginary part of the mode $V$. The quantity $V_{U}$ represents the velocity part of the mixed unknown vector $V$.

\subsection{Newton method}

The previous nonlinear system of Equation (4) can be written in the following form:

$$
\left\{\begin{aligned}
\mathrm{R}(\Lambda)=0 & \text { in } \Omega \\
V_{U}=0 & \text { on } \partial_{U} \Omega
\end{aligned}\right.
$$

where $\Lambda$ is the unknown vector. This solution is sought under the following expression:

$$
\Lambda=\Lambda_{0}+\Delta \Lambda
$$

$\Lambda_{0}$ is the given initial value, and $\Delta \Lambda$ is the unknown increment. By introducing the previous relation into Equation (5) and by neglecting the second order term in $\Delta \Lambda$, one obtains the linear system to be solved at each iteration:

$$
\left\{\begin{aligned}
\mathrm{L}_{\mathrm{t}}^{\Lambda_{0}}(\Delta \Lambda) & =-\mathrm{R}\left(\Lambda_{0}\right) & & \text { in } \Omega \\
V_{U} & =0 & & \text { on } \partial_{U} \Omega
\end{aligned}\right.
$$

where $\mathrm{R}\left(\Lambda_{0}\right)$ is the residual vector computed at the initial value $\Lambda_{0}$ (i.e. $\Lambda_{0}$ is introduced in expressions (5)) and $\mathrm{L}_{\mathrm{t}}^{\Lambda_{0}}(\Delta \Lambda)$ is the tangent operator computed at the initial point $\Lambda_{0}$. To obtain the same number of equations and unknowns, two additional conditions are introduced, which give a well posed problem (see $[3,4,11])$. Finally, finding a Hopf bifurcation point consists in determining iteratively solution $\Lambda$ which verifies:

$$
\|\mathrm{R}(\Lambda)\|<\varepsilon
$$

where $\|\bullet\|$ is the Euclidian norm of vector $\bullet$, and $\varepsilon$ is a chosen accuracy parameter. In the numerical tests studied, the value of this parameter is set and equal to $10^{-8}$. As in reference [1], choosing as initial value $\Lambda_{0}$ of the quantities coming from the computation of a bifurcation indicator is proposed.

\subsection{Bifurcation indicator}

In reference [2], the authors have proposed to determine a Hopf bifurcation by introducing an indicator. This indicator was first proposed in references $[12,13]$ in a solid mechanics framework.

In this method, the steady solution $U^{S}$ is computed independently by means of an asymptotic numerical method $([8,14,15])$. The determination of pulsation $\omega$ and mode $V$ is then solved by introducing an indicator $\phi$ in Equation (4), where the quantities $\left(U^{S}, \lambda^{S}\right)$ are known. This indicator is a scalar function, which has the properties to be always positive and null at the Hopf bifurcation 
points [12]. Considering that the steady solution is known and Equation (4) haS been rewritten with the indicator $\phi$, the following problem has to be solved:

$$
\left\{\begin{array}{cc}
i \omega M(V)+L(V)+\underset{\left(V, U^{s}\right)+Q\left(U^{s}, V\right)=\phi f}{Q} & \text { in } \Omega \\
V_{U}=0 & \text { on } \partial_{U} \Omega
\end{array}\right.
$$

where $f$ is a known random vector. This problem is solved by using the finite element method. The following discrete problem has to be solved:

$$
\left\{\left[\begin{array}{cc}
K_{t} & -\omega M \\
\omega M & K_{t}
\end{array}\right]_{V_{U}=0}\left\{\begin{array}{c}
V^{a} \\
V^{b}
\end{array}\right\}=\left\{\begin{array}{c}
\phi f \\
0
\end{array}\right\} \begin{array}{c}
\text { in } \Omega \\
\text { on } \partial_{U} \Omega
\end{array}\right.
$$

where matrix $K_{t}$ is the discrete tangent operator which is defined by $\mathrm{L}_{t}(\bullet)=L(\bullet)+Q\left(\bullet, U^{S}\right)+$ $Q\left(U^{S}, \bullet\right)$. To have a well-formulated problem, a condition of normalization is imposed and has to be applied to both the real and imaginary parts of mode V. In past studies $[2,16]$, it has been shown that some conditions lead to numeric instabilities, and that quadratic conditions are the most stable and the most adapted conditions to the perturbation method. According to these studies $[2,16]$, the following quadratic condition is applied:

$$
\|V\|^{2}=\left\|V_{\text {initial }}\right\|^{2}
$$

where $V_{\text {initial }}$ is the solution of Equation (9) for $\omega=0.0$ and $\phi=1.0$. Finally, expressions (10) and (11) permit one to compute the evolution of the indicator $\phi$ versus the angular frequency for a fixed value of the stationary solution $U^{S}$. In other words, an indicator curve, $\phi_{\operatorname{Re}}(\omega)$, is computed for each Reynolds number.

This indicator can be computed by varying the value of the angular pulsation $\omega$ in Equation (10). Thus, each value of the indicator requires the triangulation of the matrix defined in Equation (10). Finally, with this method, the computational time needed for the calculus of this indicator can be large. To avoid this problem, the indicator curve is determined by using a perturbation method. Hence, only one matrix triangulation is carried out to compute an analytic part of the indicator curve.

First, the indicator is computed as a polynomial expansion of pulsation $\omega=\omega_{0}+\hat{\omega}$, where $\omega_{0}$ is the initial angular frequency and $\hat{\omega}$ is the perturbated parameter:

$$
\left\{\begin{aligned}
\phi(\hat{\omega}) & =\phi_{0}+\hat{\omega} \phi_{1}+\hat{\omega}^{2} \phi_{2}+\ldots+\hat{\omega}^{p} \phi_{p} \\
V^{a} & =V_{0}^{a}+\hat{\omega} V_{1}^{a}+\hat{\omega}^{2} V_{2}^{a}+\ldots+\hat{\omega}^{p} V_{p}^{a} \\
V^{b} & =V_{0}^{b}+\hat{\omega} V_{1}^{b}+\hat{\omega}^{2} V_{2}^{b}+\ldots+\hat{\omega}^{p} V_{p}^{b}
\end{aligned}\right.
$$

In Equations (12), $V_{0}^{a}, V_{0}^{b}$ and $\phi_{0}$ are known and regular solutions of problem (10) and $p$ is the order of truncature of the polynomial expansions.

Inserting expansion (12) in Equation (10), and balancing terms with identical powers of $\hat{\omega}$, the following set of linear equations is obtained:

Order 0:

$$
\begin{array}{ll}
K_{t}\left(V_{0}^{a}\right)-\omega_{0} M\left(V_{0}^{b}\right)=\phi_{0} f & \text { in } \Omega \\
K_{t}\left(V_{0}^{b}\right)+\omega_{0} M\left(V_{0}^{a}\right)=0 & \text { in } \Omega \\
V_{0}^{a}=V_{0}^{b}=0 & \text { on } \partial_{u} \\
\left\|V_{0}\right\|^{2}=\left\|V_{\text {initial }}\right\|^{2} &
\end{array}
$$

Order $p$ :

$$
\begin{array}{ll}
K_{t}\left(V_{p}^{a}\right)-\omega_{0} M\left(V_{p}^{b}\right)=\phi_{p} f+M\left(V_{p-1}^{b}\right) & \text { in } \Omega \\
K_{t}\left(V_{p}^{b}\right)+\omega_{0} M\left(V_{p}^{a}\right)=-M\left(V_{p-1}^{a}\right) & \text { in } \Omega \\
V_{p}^{a}=V_{p}^{b}=0 & \text { on } \partial_{u} \Omega \\
2<V_{p}, V_{0}>+\sum_{r=1}^{p-1}<V_{p-r}, V_{r}>=0 &
\end{array}
$$


Finally, the previous linear system of equations helps compute all the unknowns at each order $p$ with a single matrix triangulation and $p$ backward and forward substitutions.

Once all the terms of the polynomial expansion are determined, the latter is replaced by equivalent rational approximations, called Padé approximants first introduced in [17]:

$$
V_{\text {Padé }}(\hat{\omega})-V_{0}=\sum_{k=1}^{p-1} \frac{P_{(p-1-k)}(\hat{\omega})}{Q_{(p-1)}(\hat{\omega})} \hat{\omega}^{k} V_{k}
$$

where $P_{k}$ and $Q_{k}$ are polynoms of degree $k$. These rational approximations increase the range of validity of the polynomial approximations [1] and consequently help decrease the number of matrix triangulations required to compute the curve $\phi_{\mathrm{Re}}(\omega)$. The validity range of the Padé approximants is determined by the following expression [10]:

$$
\delta=\frac{\left\|V_{\text {Padé }, p}\left(\hat{\omega}_{\max }\right)-V_{\text {Padé, } p-1}\left(\hat{\omega}_{\max }\right)\right\|}{\left\|V_{\text {Padé }, p}\left(\hat{\omega}_{\max }\right)-V_{0}\right\|}
$$

where $\delta$ is a small chosen parameter $\left(10^{-3} \leq \delta \leq 10^{-6}\right)$. With the latter expression, one can define the maximum value of the perturbation parameter, $\hat{\omega}_{\max }$, and then compute a new starting point of the perturbation method by introducing $\hat{\omega}_{\max }$ in the asymptotic expansions (12). Finally, the indicator curve, $\phi_{R e}(\omega)$, is entirely computed by applying, in a step by step manner, the previous continuation method.

\subsection{Additional remarks}

The present method can be viewed as an eigenvalue problem. The eigenvalues, corresponding to Hopf's bifurcations, are obtained when the indicator is null.

For that kind of problem, iterative methods such as the inverse power method [18] can be used. In solid mechanics [19], it has been shown that the results obtained by an iterative method and those obtained by a perturbation method are the same. Nevertheless, it has also been shown in the latter study, that the acceleration convergence method has to be used to obtain acceptable efficiency. In the studies $[19,20]$, the convergence acceleration methods are based on the use of Padé approximants or the extrapolation method.

\section{PROPOSED ALGORITHM}

Before introducing in details the method used in this study to determine the Hopf bifurcation points, let us first give some specifications.

In reference [2], the indicator, as previously defined, was used to determine the instability points. Consequently, many computations of the indicator curves were necessary to determine the precise values of the critical Reynolds numbers and angular frequency. In [1], this indicator was associated with the Newton method. This method is denoted 'mixed method'. The indicator is first computed and gives initial values to the iterative method. These initial values are the minima of the indicator in the computed range of angular frequency. Nevertheless, the Newton method is efficient if the initial guess is not far from the solution. Thus, in some cases, the initial value does not lead to the convergence of the direct method, for example, when the Reynolds number, for which the indicator is computed, is far from the critical value. Consequently, much computation is spent in indicator calculus without giving an interesting value for the Newton method. In the results shown in [1], the indicator curves present many extrema and consequently many possible initial values for the Newton method.

Thus, in this study, we propose to determine the indicator curve $\phi_{\operatorname{Re}}=\phi_{\operatorname{Re}}(\omega)$ for a fixed Reynolds number Re and its first and second derivatives with respect to pulsation $\mathrm{d} \phi_{R e} / \mathrm{d} \omega$ and $\mathrm{d}^{2} \phi_{\mathrm{Re}} / \mathrm{d} \omega^{2}$. One has to firstly compute the roots of the first derivative and secondly check that these roots are minima of the indicator curves by introducing them in the second derivative. Therefore, these minima are determined and a set of possible candidates are easily and automatically obtained and used as initial values for the Newton procedure. 
Expression (8) allows for obtaining an analytical expression of the indicator function with respect to pulsation $\omega$. Then, the two derivative functions $d \phi / d \hat{\omega}$ and $d^{2} \phi / d \hat{\omega}^{2}$ with respect to pulsation $\hat{\omega}$ can be derived in an analytical way:

$$
\begin{aligned}
& \frac{\mathrm{d} \phi}{\mathrm{d} \hat{\omega}}(\hat{\omega})=\phi_{1}+2 \phi_{2} \hat{\omega}+3 \phi_{3} \hat{\omega}^{2}+\ldots+p \phi_{p} \hat{\omega}^{p-1} \\
& \frac{\mathrm{d}^{2} \phi}{\mathrm{d} \hat{\omega}^{2}}(\hat{\omega})=2 \phi_{2}+6 \phi_{3} \hat{\omega}+\ldots+p(p-1) \phi_{p} \hat{\omega}^{p-2}
\end{aligned}
$$

To increase the convergence, these derivative functions are also computed with the help of Padé approximants by using the two previous asymptotic expansions [17]:

$$
\begin{gathered}
\left.\frac{\mathrm{d} \phi}{\mathrm{d} \hat{\omega}}\right|_{\text {Padé }}(\hat{\omega})-\phi_{1}=\sum_{k=1}^{p-2} \frac{\bar{P}_{(p-2-k)}(\hat{\omega})}{\bar{Q}_{(p-2)}(\hat{\omega})} \hat{\omega}^{k} \phi_{k} \\
\left.\frac{\mathrm{d}^{2} \phi}{\mathrm{d} \hat{\omega}^{2}}\right|_{\text {Padé }}(\hat{\omega})-2 \phi_{2}=\sum_{k=1}^{p-3} \frac{\tilde{P}_{(p-3-k)}(\hat{\omega})}{\tilde{Q}_{(p-3)}(\hat{\omega})} \hat{\omega}^{k} \phi_{k}
\end{gathered}
$$

In expressions (19) and (20), $\bar{P}_{k}(\hat{\omega}), \bar{Q}(\hat{\omega}), \tilde{P}_{k}(\hat{\omega}), \tilde{Q}(\hat{\omega})$ appear as polynomial expressions and are combined into a rational one. All the fractions are noted to have the same respective denominator.

The roots and the poles of Padé expansions are evaluated by a classical method [21]. Among all the possible values, the minima of function $\phi(\omega)$ are extracted. The latter are the initial guesses for the Newton algorithm. The main advantage offered by the present method is to determine automatically a set of possible candidates instead of calculating a unique one which does not ensure the convergence of the Newton calculation as in reference [1].

\section{NUMERICAL RESULTS}

To highlight the performances of the proposed algorithm, we study the Hopf bifurcation points of the lid-driven cavity problem for three geometric configurations, characterized by the aspect ratio values $\mathrm{A}=0.8, \mathrm{~A}=1.0$ and $\mathrm{A}=1.5$ (Figure 1). The description of the meshes used in the numerical tests, for all the aspect ratio A, is given in Table I. For these configurations, the results issued from the literature are exposed in Table II. A particular emphasis in this section is given for the case $\mathrm{A}=1.0$.

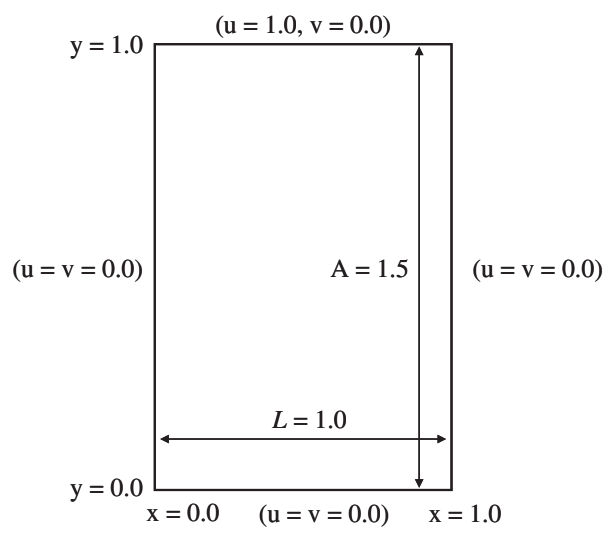

Figure 1. Geometry for the two-dimensional lid-driven cavity flow (case $A=1.5$ ). In all the calculations of the Reynolds and Strouhal numbers, the reference length is chosen as $L=1.0$. 
Table I. Characteristics of the smallest finite element used for the meshes - (R) and (I) stand respectively, for regular and irregular meshes.

\begin{tabular}{|c|c|c|c|c|c|c|c|}
\hline \multirow[b]{2}{*}{ Mesh name } & \multirow[b]{2}{*}{ D.o.F. } & \multicolumn{2}{|c|}{$A=0.8$} & \multicolumn{2}{|c|}{$\mathrm{A}=1.0$} & \multicolumn{2}{|c|}{$A=1.5$} \\
\hline & & $\Delta x$ & $\Delta y$ & $\Delta x$ & $\Delta y$ & $\Delta x$ & $\Delta y$ \\
\hline Mesh 1 & $13122(\mathrm{R})$ & 0.0250 & 0.020 & 0.0250 & 0.0250 & 0.0250 & 0.03750 \\
\hline Mesh 2 & $13122(\mathrm{I})$ & 0.0025 & 0.002 & 0.0025 & 0.0025 & 0.0025 & 0.00375 \\
\hline
\end{tabular}

Table II. Critical values for the first Hopf bifurcation in the case of the lid-driven cavity for three different geometries defined by $\mathrm{A}=1.0, \mathrm{~A}=1.5$ and $\mathrm{A}=0.8$.

\begin{tabular}{llccc}
\hline $\begin{array}{c}\text { Aspect } \\
\text { ratio, A }\end{array}$ & \multicolumn{1}{c}{ Authors } & Critical Reynolds number, & Critical Strouhal number, & Critical pulsation, \\
$\mathrm{Re}_{\mathrm{c}}$ & $\mathrm{Re}_{\mathrm{c}}$ & 2185.0 \\
$\mathrm{~A}=1.0$ & This study & 7890.0 & 0.440 & 2268.0 \\
& Boppana [4] & 8026.6 & 0.4497 & 2261.9 \\
& Fortin [5] & 8000.0 & 0.4500 & - \\
& Abouhamza [22] & 8004.5 & - & 2314.8 \\
& Tiesinga [23] & 8375.0 & 0.4399 & 2195.0 \\
& Poliashenko [26] & 7763.4 & 0.4500 & - \\
& Bruneau [27] & $8000-8050$ & - & 2744.0 \\
& Peng [28] & 7402.0 & 0.5900 & 2250.6 \\
& Gervais [29] & 7960.0 & 0.4500 & 2273.3 \\
& & 8040.0 & 0.4500 & 2996.8 \\
& Cazemier [30] & 7819.0 & 0.6100 & 2254.0 \\
& Auteri [31] & 7972.0 & 0.4500 & 2265.0 \\
& & 8018.0 & 0.4496 & 973.0 \\
$\mathrm{~A}=1.5$ & This study & & & 1137.8 \\
& Boppana [4] & 4722.0 & 0.3280 & 1746.9 \\
& Abouhamza [22] & 5326.0 & 0.3400 & 1994.9 \\
& Poliashenko [26] & 5674.0 & 0.4900 & 1152.0 \\
$\mathrm{~A}=0.8$ & This study & 7216.0 & 0.4400 & 1149.0 \\
\hline
\end{tabular}

\subsection{Results with the proposed algorithm}

In the case of a lid-driven cavity parameter $\mathrm{A}=1.0$, three initial values of Reynolds number $\mathrm{Re}_{i}$ are chosen: $\operatorname{Re}_{i}=6332,7257,8089$ (see Table III). For this example, the indicator curve $\phi(\omega)$ and the derivative functions $\mathrm{d} \phi / \mathrm{d} \omega$ and $\mathrm{d}^{2} \phi / \mathrm{d} \omega^{2}$ are plotted in Figures 2-4 for a Reynolds number equal to 6332. In the latter, the curves are exposed in a non-dimensional form using the Strouhal number $\mathrm{St}=\omega L /(2 \pi u)$. These curves are computed by using a truncature order of the asymptotic expansions equal to 20 and a parameter $\delta$ equal to $10^{-6}$. The indicator is computed by using the Padé-based continuation method up to an angular frequency equal to 5000. Let us remark that these parameters are the same for all the numerical tests presented in this work.

For each of these three chosen values, the present algorithm determines several initial values of pulsation $\omega_{i}$ and vector $V_{i}: 13,14$ and 9 initial couples $\left(\omega_{i}-V_{i}\right)$ are obtained for $\operatorname{Re}=6332,7257$ and 8089, respectively. For each Reynolds number, at least one critical Reynolds number is obtained, that is to say at least one Hopf bifurcation is determined (see results in Table III). It is considered in this study that the Newton algorithm converges if the norm of the residual vector is lower than $10^{-4}$ and $10^{-8}$, respectively, for the stationary and dynamic solution. The maximum number of iterations is set as equal to 20. When the Newton method converges to a critical value, the required number of iterations is given in Table III. Symbol '-' indicates that the iterative method does not find any critical values after 20 iterations.

However, it is noticed that the convergence to a critical number is not necessarily obtained for the minimum value of indicator $\phi_{i}$. This is a noticeable fact and constitutes a major feature compared with the previous work [1], where all the calculations are based on the values obtained for the 


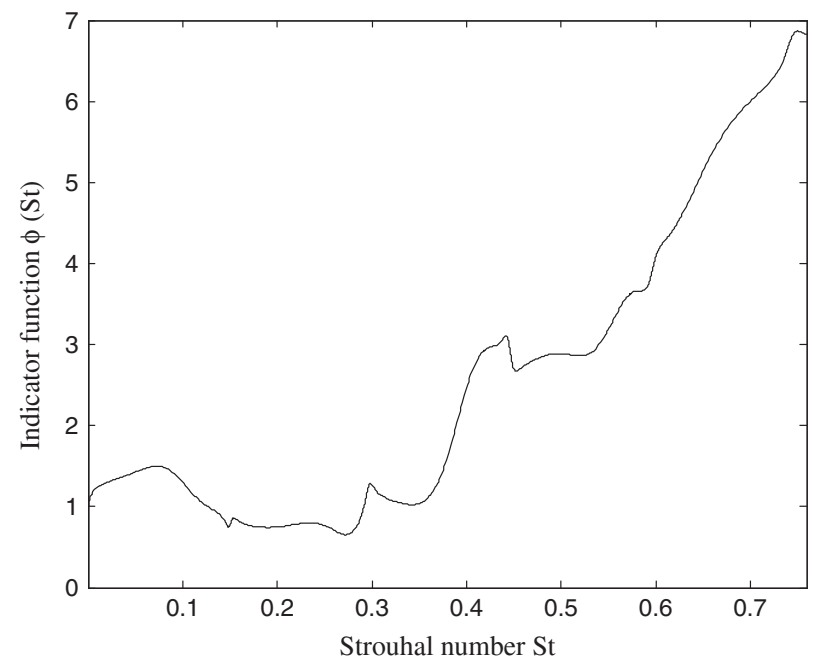

Figure 2. Evolution of the indicator function with respect to the Strouhal number St-Problem of the liddriven cavity-Case $\mathrm{A}=1.0$ for $\mathrm{Re}=6332$. The truncature order of asymptotic equations and the parameter $\delta$ are, respectively, equal to 20 and $10^{-6}$.

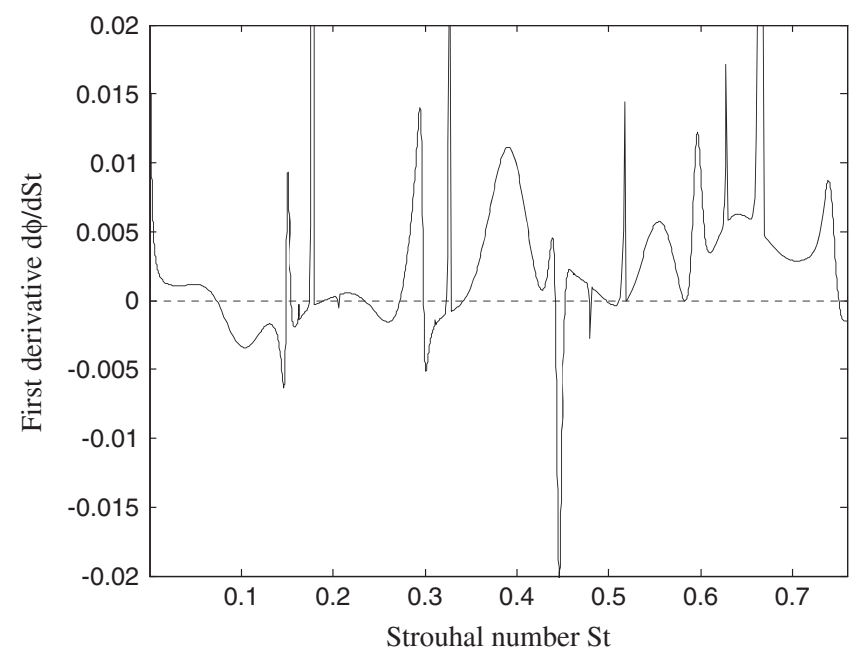

Figure 3. Evolution of the first derivative function of the indicator with respect to the Strouhal Number StProblem of the lid-driven cavity-Case $\mathrm{A}=1.0$ for $\mathrm{Re}=6332$. The truncature order of asymptotic equations and the parameter $\delta$ are, respectively, equal to 20 and $10^{-6}$.

minimum of the indicator $\phi_{i}$. In other words, according to the previous algorithm [1] and by considering the case $\operatorname{Re}=6332$, all the calculations with the retained values $\omega_{i}=1085$ and $\phi_{i}=0.653$ were unnecessary, as convergence was not obtained for this set of values. This is also the case when the considered initial Reynolds number is equal to 7257, and this can be noted as one of the drawbacks of the previously proposed algorithm [1].

The minimum value of the indicator is then not a sufficient condition to ensure the algorithm convergence. Thus, the present algorithm offers the real improvement of having a set of candidates to calculate Hopf bifurcation points. For example, six, five and five different critical points are found with an initial Reynolds number respectively equal to 6332, 7257 and 8089.

To verify that the bifurcation indicator gives good initial values, we define the following correlation coefficient:

$$
\mathrm{C}\left(V_{i}, V_{c}\right)=\frac{<V_{i}, V_{c}>}{\left\|V_{i}\right\| \cdot\left\|V_{c}\right\|}
$$




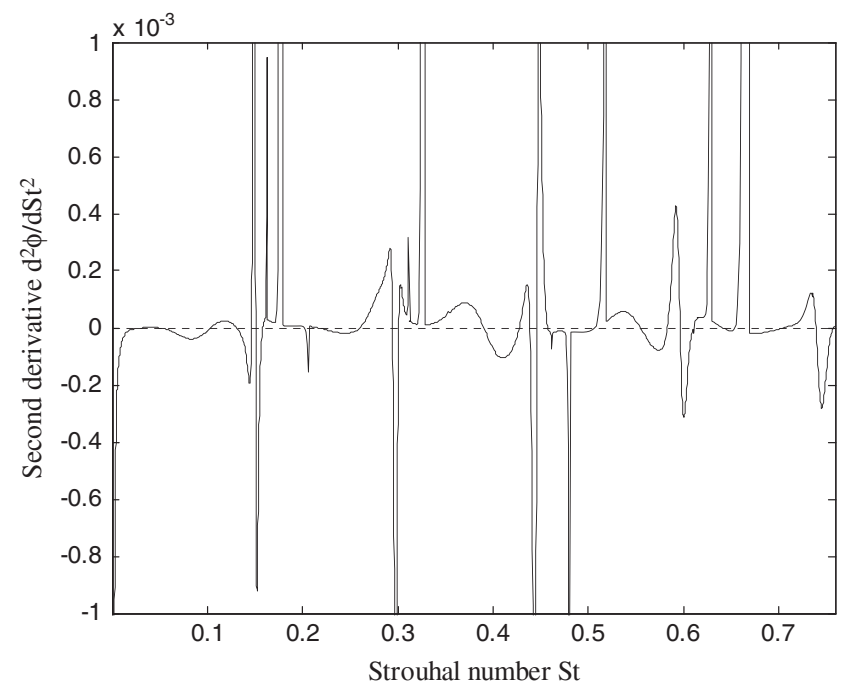

Figure 4. Evolution of the second derivative function of the indicator with respect to the Strouhal Number $\mathrm{St}$-Problem of the lid-driven cavity-Case $\mathrm{A}=1.0$ for $\mathrm{Re}=6332$. The truncature order of asymptotic equations and the parameter $\delta$ are, respectively, equal to 20 and $10^{-6}$.

where vector $V_{i}$ is the initial velocity (computed from the minimum value of the indicator) and $V_{c}$ is the critical Hopf mode found. For example, if one considers the results obtained in the case $\mathrm{Re}_{\mathrm{i}}=8089$ (Table III), the proposed algorithm gives six initial values for the Newton method. Among these values, from the couple $\left(\omega_{\mathrm{i}}=2236, \phi_{i}=0.395\right)$ four Newton iterations are needed to obtain the first Hopf bifurcation point (i.e. $\operatorname{Re}_{\mathrm{c}}=7890, \omega_{c}=2185$ ). Thus, if this initial vector is introduced in the correlation coefficient as $V_{i}$ and the computed first bifurcation vector as $V_{c}$, this correlation coefficient is then equal to 0.97 (see Table IV), which is very close to 1 . In Table IV, for all the computed Hopf points, this correlation coefficient is given and is never lower than 0.6 even for critical Reynolds numbers far from the initial value. For example, for the critical Reynolds number equal to $\mathrm{Re}_{\mathrm{c}}=11418$ and $\omega_{\mathrm{c}}=5637$, this correlation coefficient is equal to 0.67 .

The results obtained from the proposed algorithm are very close to the critical values issued from the literature for the first bifurcation point $\left(\operatorname{Re}_{\mathrm{c}}=7890, \omega_{\mathrm{c}}=2185\right)$. The latter are summarized in Table II. Indeed, for a geometric aspect ratio $\mathrm{A}=1.0$, the critical Reynolds number is between 7400 , and 8050 and the Strouhal number is close to 0.44 .

The values exposed in Table III are directly issued from the calculations. Surprisingly, it can be observed that the critical points are not found in function with the increasing Reynolds number. The algorithm seems to compute the solutions in function with the increasing Strouhal numbers.

The present method is designed to offer an automatic algorithm to compute Hopf bifurcation points. The results exposed in Table $\mathrm{V}$ show that the algorithm is able to find, automatically, 10 critical values declared as Hopf bifurcation without any user intervention. This is an advantageous characteristic for parametric study, for which many calculations are required. It can be noticed that the fundamental Hopf bifurcation is also obtained. These 10 critical values are obtained by computing the indicator curves for some Reynolds numbers in the range [2000-16000]. The streamline real and imaginary vectors of these Hopf bifurcation points are plotted respectively in Figures 5 and 6. For these plots, we only consider the first five critical values. For the first Hopf bifurcation, $\mathrm{Re}_{\mathrm{c}}=7890$ and $\mathrm{St}_{\mathrm{c}}=0.44$, one can remark that these streamlines are quite similar to those shown in references $[4,5,22]$.

Thus in this range, the performance of the presented algorithm is evaluated through Figure 7 and 8. Figure 7 presents the evolution of the number of roots and bifurcation points in function with the Reynolds number in the case of the lid-driven cavity with $A=1.0$. It can be seen that the number of possible candidates is comprised between 0 and 16 for the Reynolds numbers tested. Moreover, 
Table III. Numerical results obtained for the problem of the lid-driven cavity — case A=1.0 - Critical Reynolds and Strouhal numbers obtained for initial Reynolds and Strouhal numbers evaluated as minima of the indicator curve. Symbol '-' stands for the non-convergence of the calculus.

\begin{tabular}{|c|c|c|c|c|c|c|}
\hline \multicolumn{3}{|c|}{ Initial values } & \multicolumn{4}{|c|}{ Critical values } \\
\hline $\begin{array}{l}\text { Reynolds } \\
\text { number, } \operatorname{Re}_{\mathrm{i}}\end{array}$ & $\begin{array}{l}\text { Pulsation, } \\
\qquad \omega_{i}\end{array}$ & $\begin{array}{c}\text { Indicator, } \\
\phi_{\mathrm{i}}\end{array}$ & $\begin{array}{l}\text { Reynolds number, } \\
\qquad \operatorname{Re}_{\mathrm{c}}\end{array}$ & $\begin{array}{l}\text { Pulsation, } \\
\omega_{\mathrm{c}}\end{array}$ & $\begin{array}{c}\text { Strouhal number, } \\
\mathrm{St}_{\mathrm{c}}\end{array}$ & $\begin{array}{c}\text { Number of } \\
\text { iterations }\end{array}$ \\
\hline \multirow[t]{13}{*}{6332} & 591 & 0.745 & - & - & - & - \\
\hline & 757 & 0.744 & - & - & - & - \\
\hline & 1085 & 0.653 & - & - & - & - \\
\hline & 1361 & 1.020 & 11286 & 2385 & 0.33 & 9 \\
\hline & 1799 & 2.670 & 7890 & 2185 & 0.44 & 6 \\
\hline & 2066 & 2.860 & 8829 & 2925 & 0.52 & 8 \\
\hline & 2159 & 3.660 & 8829 & 2925 & 0.52 & 8 \\
\hline & 2323 & 5.700 & 8921 & 3424 & 0.61 & 10 \\
\hline & 3239 & 6.680 & 11417 & 5636 & 0.78 & 8 \\
\hline & 3450 & 6.940 & - & - & - & - \\
\hline & 3552 & 6.830 & 12625 & 6877 & 0.87 & 16 \\
\hline & 3961 & 6.560 & - & - & - & - \\
\hline & 4515 & 9.030 & - & - & - & - \\
\hline \multirow[t]{14}{*}{7257} & 677 & 0.654 & - & - & - & - \\
\hline & 925 & 0.667 & - & - & - & - \\
\hline & 1231 & 0.508 & - & - & - & - \\
\hline & 1582 & 0.699 & 11286 & 2385 & 0.33 & 6 \\
\hline & 2021 & 1.300 & 7890 & 2185 & 0.44 & 5 \\
\hline & 2418 & 1.610 & 8829 & 2925 & 0.52 & 7 \\
\hline & 2701 & 2.750 & - & - & - & - \\
\hline & 2776 & 2.660 & 8921 & 3424 & 0.61 & 6 \\
\hline & 3216 & 4.850 & - & - & - & - \\
\hline & 3701 & 5.740 & - & - & - & - \\
\hline & 3759 & 4.580 & - & - & - & - \\
\hline & 4075 & 6.110 & - & - & - & - \\
\hline & 4159 & 5.690 & 12625 & 6877 & 0.87 & 10 \\
\hline & 4502 & 6.220 & - & - & - & - \\
\hline \multirow[t]{9}{*}{8089} & 754 & 0.640 & - & - & - & - \\
\hline & 1085 & 0.606 & - & - & - & - \\
\hline & 1360 & 0.418 & - & - & - & - \\
\hline & 1759 & 0.486 & 11286 & 2385 & 0.33 & 6 \\
\hline & 2236 & 0.395 & 7890 & 2185 & 0.44 & 4 \\
\hline & 2691 & 0.669 & 8829 & 2925 & 0.52 & 5 \\
\hline & 3105 & 1.230 & 8921 & 3424 & 0.61 & 5 \\
\hline & 3614 & 3.120 & 9513 & 4226 & 0.70 & 7 \\
\hline & 4100 & 4.630 & 11417 & 5636 & 0.78 & 9 \\
\hline
\end{tabular}

Table IV. Example of the lid-driven cavity, value of the correlation number $C$. The initial Reynolds number is equal to 8089 .

\begin{tabular}{lccc}
\hline Initial pulsation, $\omega_{\mathrm{i}}$ & Critical pulsation, $\omega_{\mathrm{c}}$ & Critical Reynolds number, $\mathrm{Re}_{\mathrm{c}}$ & Correlation factor, $C\left(V_{\mathrm{i}}, V_{\mathrm{c}}\right)$ \\
\hline 2236 & 2186 & 7890 & 0.97 \\
3105 & 3424 & 8921 & 0.91 \\
3614 & 4226 & 9513 & 0.87 \\
1759 & 2386 & 11287 & 0.86 \\
2961 & 2925 & 8829 & 0.85 \\
4100 & 5637 & 11418 & 0.67 \\
\hline
\end{tabular}

no matter the initial Reynolds number included in the range [3000-14 000], at least one bifurcation point is always computed. For example, for a Reynolds number equal to $\operatorname{Re} \approx 6200$, the algorithm retains 16 initial values $R_{i}$ and computes six critical Reynolds numbers $R_{c}$. 
Table V. Example of the lid-driven cavity-case $A=1.0$ - Presentation of the 10 computed critical Reynolds and Strouhal numbers.

\begin{tabular}{lcc}
\hline Hopf bifurcation number & Critical Reynolds number, $\mathrm{Re}_{\mathrm{c}}$ & Critical Strouhal number, St \\
\hline 1 & 7890 & 0.44 \\
2 & 8829 & 0.52 \\
3 & 8921 & 0.61 \\
4 & 9513 & 0.71 \\
5 & 11286 & 0.33 \\
6 & 11417 & 0.78 \\
7 & 12625 & 0.86 \\
8 & 14674 & 0.92 \\
9 & 15501 & 0.99 \\
10 & 16170 & 0.24 \\
\hline
\end{tabular}

Figure 8 exposes the rank of the critical points in function with the initial Reynolds number $\mathrm{Re}_{\mathrm{i}}$. This rank is given in Table V. For example, it can be observed that the fundamental Hopf Bifurcation (point $n^{\circ} 1$ ) is computed from 13 previously isolated initial Reynolds numbers $\operatorname{Re}_{i}$. It can be noted that the convergence range is significant: the fundamental Hopf bifurcation point is calculated for initial Reynolds number $\mathrm{Re}_{\mathrm{i}}$ comprised between $[\approx 3500-\approx 9500]$. For information, it can be noticed that a $10^{\text {th }}$ critical point can be computed for high values of initial Reynolds numbers. Finally, it is observed that the Hopf bifurcation $n^{\circ} 5$, corresponding to a minimum Strouhal number $\left(\mathrm{St}_{\mathrm{c}}=0.33\right)$ is found 22 times in a large range of Reynolds number. Hence, the proposed algorithm gives many possible Hopf bifurcation points. In the literature, few references give critical parameters about the second or third instability point. Nevertheless, in reference [23], the authors, by computing the eigenvalues of the Jacobian matrix, give an approximation of the first critical points. These authors have found that the Hopf bifurcation points appear for the following Reynolds number $\mathrm{Re}_{\mathrm{c} 1} \in$ [8000-8375], $\mathrm{Re}_{\mathrm{c} 2} \in$ [8375-8875], $\operatorname{Re}_{\mathrm{c} 3} \in$ [8875-9375], $\mathrm{Re}_{\mathrm{c} 4} \in$ [9875-10375] and respectively for the following Strouhal numbers: 0.44, 0.6, 0.52, 0.7 and 0.77. A quick comparison between the latter results and ours shows that the Strouhal numbers are the same. Indeed, results in Table III show that we found exactly these five Strouhal numbers among the first six bifurcation points. Nevertheless, these numbers are not found for the same critical Reynolds numbers in both studies. For example, in reference [23], they have found the first bifurcation for a Reynolds number between [8000-8375] $\left(\operatorname{Re}_{\mathrm{c} 1}=7890\right.$ in this study). Moreover, in this reference, the second Hopf bifurcation point is obtained for a Reynolds number included in [8375-8875] and for a Strouhal number equal to 0.6. It seems that this point does not correspond to the second, but to the third bifurcation point we found (see Table V). The second and third Hopf bifurcation points are then reversed compared with those computed in reference [23]. This dissimilarity can be explained by the fact that both studies use different numerical schemes. For example, the space discretization is performed with a finite volume method in reference [23], whereas in this study, a finite element method is used.

\subsection{Comparison with other numerical methods}

In this section, we compare the critical values obtained with four numerical methods. The first one is the initial bifurcation indicator presented in [2], the second one is the hybrid method [1], the third one is the proposed method and the last one consists in computing the eigenvalues of the Jacobian matrix for a chosen Reynolds number and in checking if a pair of eigenvalues crosses the imaginary axis.

The computation of the eigenvalues of the Jacobian matrix allows the bifurcation points to be determined. Hence, a Hopf bifurcation is characterized by two eigenvalues, which are purely imaginary. However, the Jacobian matrix is non symmetric, and specific algorithms have to be used. In this paper, we propose comparing the critical Reynolds numbers obtained with the proposed algorithm and those calculated with the ARPACK [24] package based on the Arnoldi method [25]. The results obtained from the eigenvalue calculations for different Reynolds numbers are exposed in 


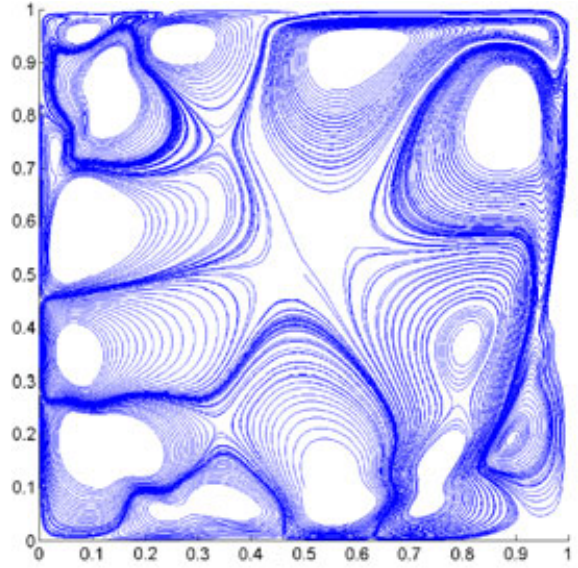

(a) $\mathrm{Re}_{\mathrm{c}}=7890, \mathrm{St}_{\mathrm{c}}=0.44$

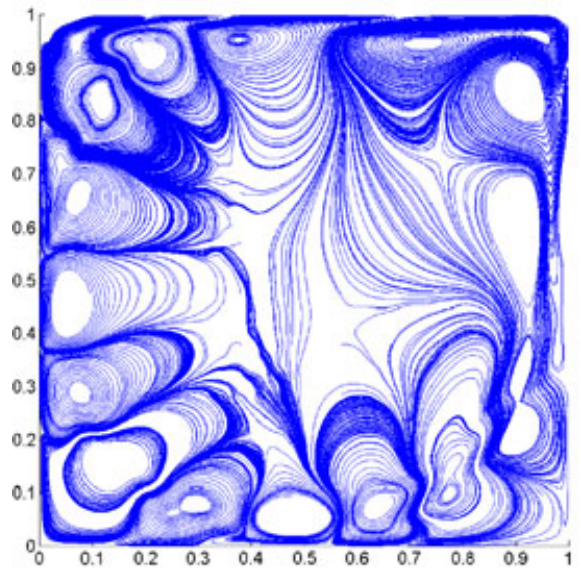

(c) $\mathrm{Re}_{\mathrm{c}}=8921, \mathrm{St}_{\mathrm{c}}=0.61$

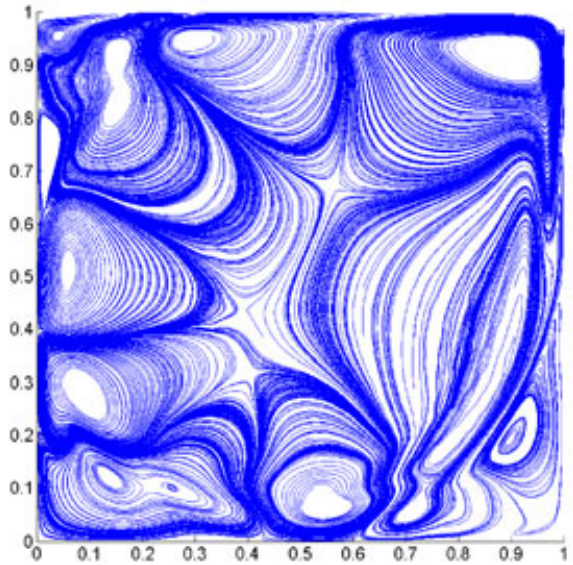

(e) $\operatorname{Re}_{\mathrm{c}}=11286, \mathrm{St}_{\mathrm{c}}=0.33$

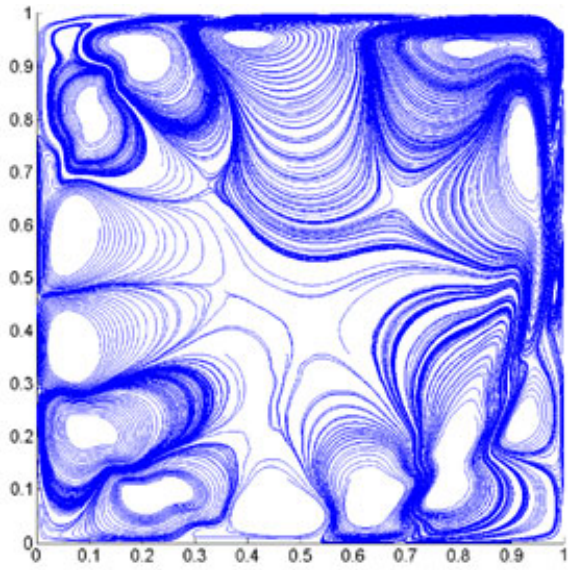

(b) $\mathrm{Re}_{\mathrm{c}}=8829, \mathrm{St}_{\mathrm{c}}=0.52$

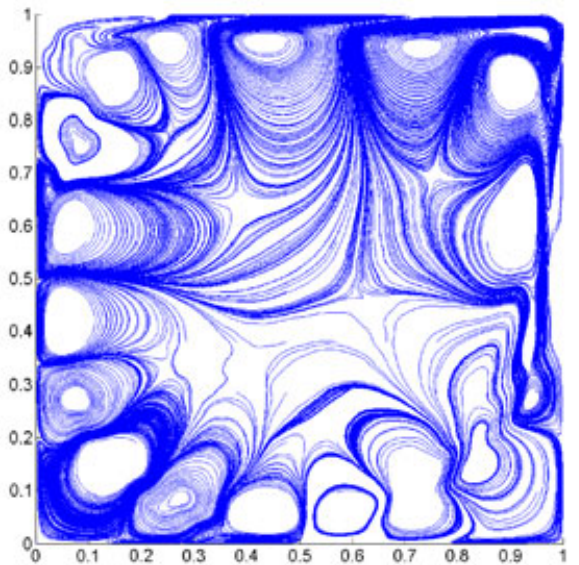

(d) $\mathrm{Re}_{\mathrm{c}}=9513, \mathrm{St}_{\mathrm{c}}=0.71$

Figure 5. Streamlines of the real part of the eigenvectors for several critical Reynolds numbers. The lid-driven cavity $\mathrm{A}=1.0$.

Figure (9), for the lid-driven cavity with an aspect ratio equal to 1 (Mesh 2, 13122 D.o.F.). The evolution of the eigenvalues versus the Reynolds number is quite similar to Figure 4 of reference [23]. A comparison of the results obtained with the eigenvalue computations with the critical parameters found with the proposed algorithm is presented in Table VI. The relative percentage error is quite 


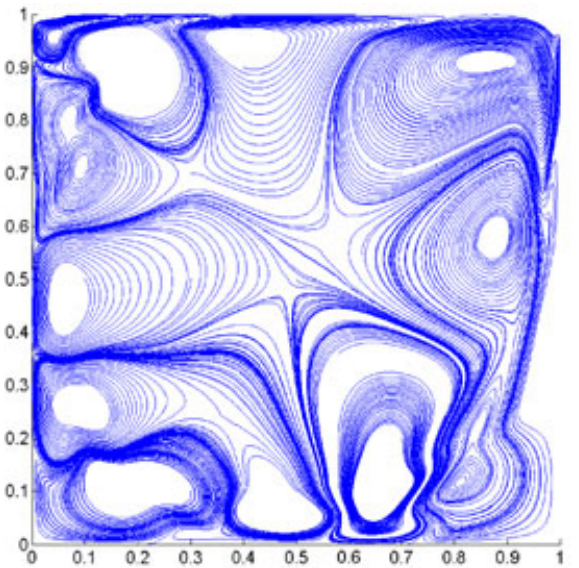

(a) $\mathrm{Re}_{\mathrm{c}}=7890, \mathrm{St}_{\mathrm{c}}=0.44$

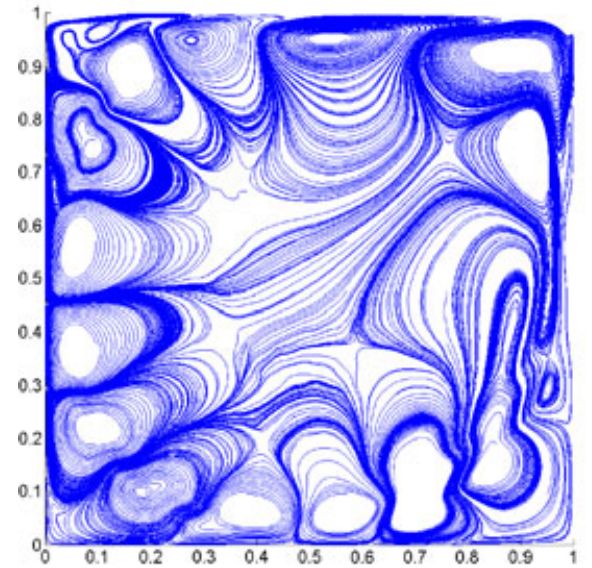

(c) $\mathrm{Re}_{\mathrm{c}}=8921, \mathrm{St}_{\mathrm{c}}=0.61$

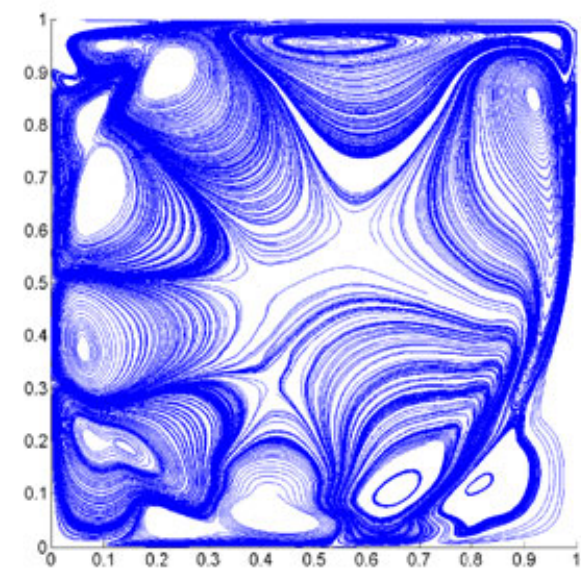

(e) $\mathrm{Re}_{\mathrm{c}}=11286, \mathrm{St}_{\mathrm{c}}=0.33$

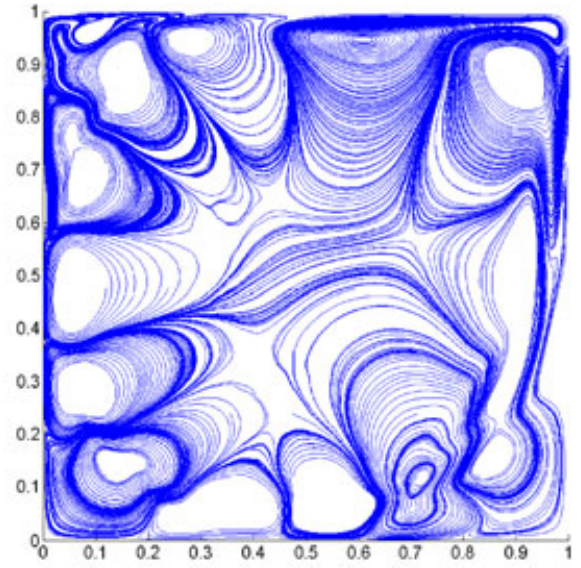

(b) $\mathrm{Re}_{\mathrm{c}}=8829, \mathrm{St}_{\mathrm{c}}=0.52$

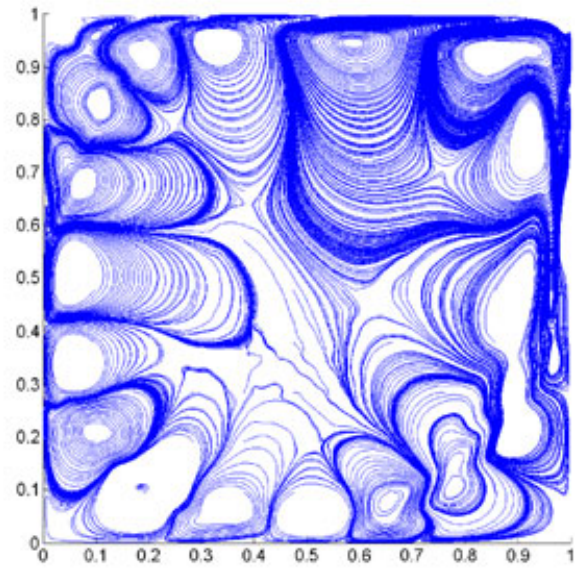

(d) $\mathrm{Re}_{\mathrm{c}}=9513, \mathrm{St}_{\mathrm{c}}=0.71$

Figure 6. Streamlines of the imaginary part of the eigenvectors for several critical Reynolds numbers. The lid-driven cavity $\mathrm{A}=1.0$.

small. This indicates that for the numerical scheme used in this study, the critical parameters found with the proposed method seem to be correct.

We now compare the computational times required for the four considered methods to determine Hopf bifurcation. It is quite difficult to seriously compare these four methods. Indeed, two methods 


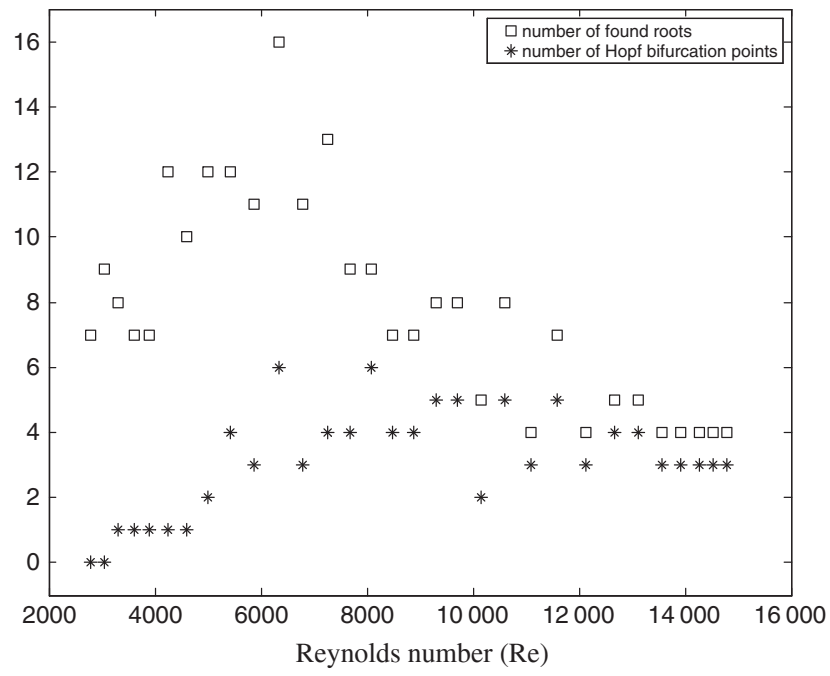

Figure 7. Number of computed roots and determined bifurcation points for different Reynolds numbersExample of the lid-driven cavity-Case A = 1.0 - Mesh 1.

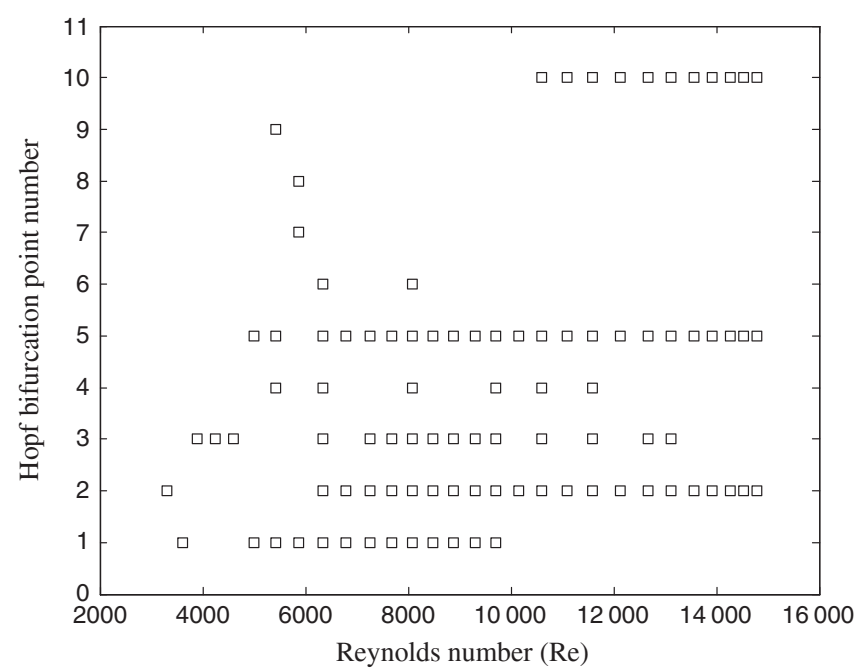

Figure 8. Results of the Hopf bifurcation number for different initial Reynolds numbers (related to the Table V)—Example of the lid-driven cavity_Case A = 1.0 - Mesh 1.

give accurate values of the Hopf bifurcation points (the Hybrid and the proposed method), and the two others only give approximate values of the critical parameters (the bifurcation indicator and ARPACK [24]). Nevertheless, we give hereafter some elements, which permit us to exhibit the advantages and drawbacks of each method.

Thus, in Table VII, the basic CPU times are given for three methods to compute Hopf bifurcation points in the case of the lid-driven problem with an aspect ratio $A=1.0$ and a number of D.o.F. equal to 13222 .

The first method is the indicator presented in [2]. With this method, 66 steps are necessary to obtain the indicator curve in the range $\omega \in[0,5000]$. Each step requires a computational time of nearly $68 \mathrm{~s}$ (according to the results presented in reference [1]). Finally, for each initial Reynolds number, the indicator curve is determined with nearly $4500 \mathrm{~s}$. In Table VII, the computational time for a single Newton iteration is also given. This time is equal to $60 \mathrm{~s}$. Finally, the computational time required with ARPACK [24] to give a part of the spectrum is also indicated in this table. This computational time is approximatively $1440 \mathrm{~s}$. 


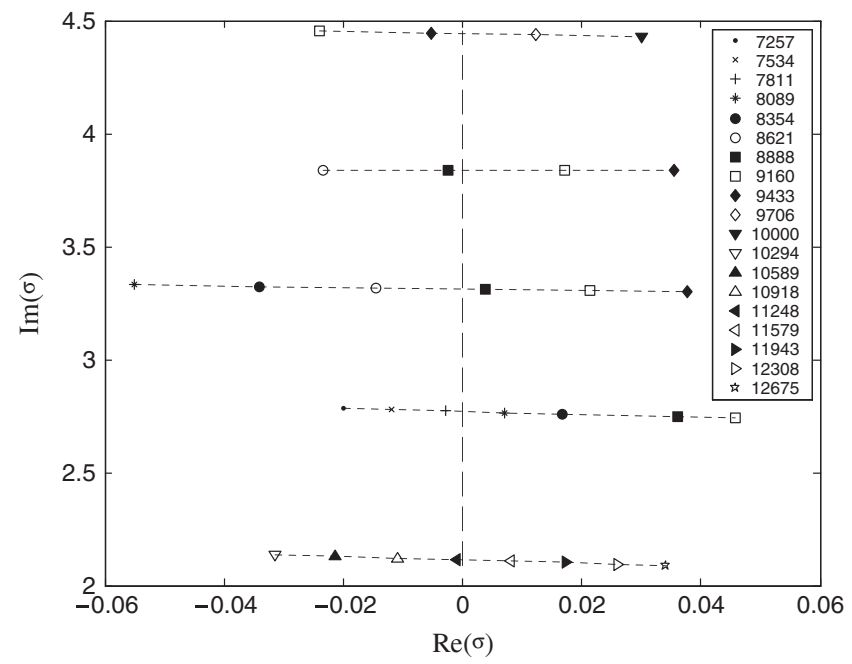

Figure 9. Representation in a complex plane of the eigenvalues of the Jacobian matrix for 19 different values of the Reynolds number. Symbol $\sigma$ stands for the complex eigenvalue of the tangent operator $K_{T}$. Estimation of five critical pulsations corresponding to $\operatorname{Re}(\sigma)=0: \omega_{\mathrm{c} 1}=2183, \omega_{\mathrm{c} 2}=2387, \omega_{\mathrm{c} 3}=2926, \omega_{\mathrm{c} 4}=3424$, $\omega_{\mathrm{c} 5}=4226$-Example of the lid-driven cavity $(\mathrm{A}=1.0)$.

Table VI. Comparison of the critical pulsations obtained by the present algorithm with those obtained by an eigenvalue calculation (Arnoldi method)_Example of the lid-driven cavity_Case A=1.0 - Mesh 1.

\begin{tabular}{lcc}
\hline $\begin{array}{l}\text { Critical pulsation, } \omega_{\mathrm{c}}^{\mathrm{AL}} \text { obtained } \\
\text { for the present algorithm }\end{array}$ & $\begin{array}{c}\text { Critical pulsation, } \omega_{\mathrm{c}}^{\mathrm{AR}} \text { obtained } \\
\text { for the Arnoldi method }\end{array}$ & $\begin{array}{c}\text { Relative error calculated as } 100 \\
\times \quad\left|\omega_{\mathrm{c}}^{\mathrm{AL}}-\omega_{\mathrm{c}}^{\mathrm{AR}}\right| / \omega_{\mathrm{c}}^{\mathrm{AL}}\end{array}$ \\
\hline 2181 & 2183 & 0.08 \\
2340 & 2387 & 2.00 \\
2885 & 2926 & 1.43 \\
3419 & 3424 & 0.14 \\
\hline
\end{tabular}

Table VII. Basic central processing unit times for three methods; The considered example is the lid-driven cavity with $\mathrm{A}=1.0$ and 13122 D.o.F. (Mesh 2). The initial Reynolds number is equal to 8089.

\begin{tabular}{lc}
\hline Method & \multicolumn{1}{c}{ CPU times (s) } \\
\hline Indicator [2] for $\omega \in[0,5000]$ & $66 \mathrm{steps} \times 68 \mathrm{~s}=4500 \mathrm{~s}$ \\
Newton's iteration & $60 \mathrm{~s}$ \\
Arnoldi computation [24] & $1440 \mathrm{~s}$ \\
\hline
\end{tabular}

With these basic times, we are able to give an estimation of the CPU times needed to compute Hopf bifurcation points with the four considered methods.

Therefore, in Table VIII, these estimated CPU times are given to obtain the Hopf bifurcation point with the Hybrid method [1], the bifurcation indicator [2], an eigenvalue computation with ARPACK [24] and the proposed method. For example, the bifurcation indicator [2] requires at least three computations to check if a Hopf bifurcation occurs. By taking into account the basic CPU times given in Table VII, this leads to an approximated computational time equal to $13500 \mathrm{~s}$. Nevertheless, these three indicator curves are not sufficient to give an accurate value of the critical point. For this, additional computations are necessary and consequently the CPU times will increase. That is also the case with ARPACK [24], where more than one eigenvalue computation is required to give accurate critical parameters. Nevertheless, a single one helps certify if a Hopf bifurcation occurs by checking if a pair of conjugated eigenvalues crosses the imaginary axis. For these reasons, we have indicated in Table VIII that with the two previous methods, only estimated critical points can be found. Contrarily, with the two other methods, the Hybrid [1] and the proposed method, accurate 
Table VIII. Estimated computational times for four numerical methods to detect a Hopf bifurcation point. For the first three methods, the considered central processing unit times are issued from Table VII. The considered example is the lid-driven cavity with $\mathrm{A}=1.0$ and 13122 D.o.F. (Mesh 2). The initial Reynolds number is $\operatorname{Re}=8089$.

\begin{tabular}{llcc}
\hline Method & \multicolumn{1}{c}{ CPU time, (s) } & Number of critical values found & Critical point \\
\hline Hybrid method [1] & $4500+4 \times 60=4740$ & 1 & accurate \\
Bifurcation indicator [2] & $4500 \times 3=13500$ & 1 & estimated \\
ARPACK [24] & 1440 & 1 & estimated \\
Proposed method & $4500+36 \times 60=6660$ & 6 & accurate \\
\hline
\end{tabular}

critical parameters can be determined. Hence, the Hybrid method requires approximately $4740 \mathrm{~s}$ to determine a Hopf bifurcation point with an initial Reynolds number equal to 8089. This computational time has been evaluated by taking into account an indicator calculus (4500 s) and four iterations of Newton's method (according to the results presented in reference [1]). The proposed method requires a computational time equal to $6660 \mathrm{~s}$ which is determined by also considering an indicator calculus (4500 s) and 36 iterations of Newton's method. These 36 iterations are issued from the results presented in Table III by only taking into account the initial guesses leading to a bifurcation point. This means that, at the end of the computations, six Hopf bifurcation points are accurately determined with the proposed method instead of a single one with the Hybrid method [1].

Finally, one can conclude that with the proposed method and for the considered numerical examples, one can determine six bifurcation points with a computational time, which is approximately equal to five eigenvalue calculi with ARPACK [24]. Let us remark that to compute the same number of critical values with ARPACK [24], more than five eigenvalue computations would certainly be necessary.

The computational times of the hybrid and the proposed methods show that it is the indicator phase, which is the step that consumes the most amount of time. This topic will be dealt with in further works, which means a decrease of the CPU times required to obtain the initial guesses of Newton's method with the indicator.

\subsection{Supplementary aspect ratios}

One considers now the lid-driven cavity with two other aspect ratios: $\mathrm{A}=0.8$ and $\mathrm{A}=1.5$. For these two examples, we give, in Table II, the first Hopf bifurcation point determined by the proposed method and the ones reported in the literature. For the case $\mathrm{A}=0.8$, with the proposed method, the first bifurcation point appears for a Reynolds number and a Strouhal number respectively equal to 5297 and 0.346. These numbers are relatively close to the ones reported by Poliashenko and Aidun [26], which are respectively 5225 and 0.35 . Let us remark that these authors also use a Newton method to detect instabilities, but by taking as initial guesses the quantities resulting from a time-dependent Navier-Stokes computation. Concerning the geometric ratio $\mathrm{A}=1.5$, the results determined with the proposed method are close to the results reported by Boppana and Gajjar [4] essentially for the Strouhal number ( 0.328 for the proposed method and 0.34 in reference [4]). The critical Reynolds numbers are quite different (4722 for this study and 5326 for reference [4]). Nevertheless, comparison with the results given in references [22] and [26] shows that for this example, the critical numbers for which the first Hopf bifurcation appears is still an open question.

As with the case $\mathrm{A}=1.0$, the proposed method can give more than one bifurcation point. Thus, in Tables IX and $\mathrm{X}$, we give the first seven critical values respectively for $\mathrm{A}=0.8$ and $\mathrm{A}=1.5$. These values are also obtained by computing a single indicator curve and by determining along this curve the minima of the indicator. These values are then introduced as previously performed in the Newton scheme.

\section{CONCLUSION}

In this article, an extension of a previous study is presented [1]. The exposed algorithm allows for the automatic determination of bifurcation points by computing an indicator curve and its first 
Table IX. Presentation of the critical parameters obtained for the lid-driven cavity - Case $\mathrm{A}=0.8$-Mesh 2 .

\begin{tabular}{lcc}
\hline Critical Reynolds number, $\mathrm{Re}_{\mathrm{c}}$ & Critical pulsation, $\omega_{\mathrm{c}}$ & Critical Strouhal number, $\mathrm{St}_{\mathrm{c}}$ \\
\hline 5297 & 1152 & 0.346 \\
5790 & 1704 & 0.468 \\
5966 & 2208 & 0.589 \\
6951 & 3143 & 0.720 \\
8095 & 4250 & 0.836 \\
8934 & 5358 & 0.954 \\
10541 & 7101 & 1.072 \\
\hline
\end{tabular}

Table X. Presentation of the critical parameters obtained for the lid-driven cavity-Case $\mathrm{A}=1.5-$ Mesh 2 .

\begin{tabular}{lcc}
\hline Critical Reynolds number $\mathrm{Re}_{\mathrm{c}}$ & Critical pulsation, $\omega_{\mathrm{c}}$ & Critical Strouhal number, $\mathrm{St}_{\mathrm{c}}$ \\
\hline 4722 & 973 & 0.328 \\
6465 & 1715 & 0.422 \\
7032 & 2150 & 0.486 \\
7369 & 2656 & 0.573 \\
7903 & 3267 & 0.657 \\
8331 & 3928 & 0.750 \\
9783 & 5142 & 0.836 \\
\hline
\end{tabular}

two derivatives with respect to the pulsation of the flow $\omega$. With this method, several frequencies are computed as roots of the indicator curve and are used as initial values for an iterative Newton algorithm. The issued solution corresponds to a bifurcation point. The results exposed in this paper show the efficiency and the accuracy of the studied algorithm. Compared with the previous work, the range for the stationary solution for which Hopf bifurcation points are found is considerably increased. Thus, for the lid-driven cavity with an aspect ratio equal to 1, the first bifurcations are found from an initial Reynolds number close to 3000 . Whereas, with the hybrid method proposed in reference [1], this number is nearly 7600. The advantage of the proposed method compared, for example, with an eigenvalue computation is that the bifurcation point is automatically computed without any user intervention.

In this study, initial values are obtained using a perturbation method and transmitted to a Newton's algorithm. It could be interesting to develop a new algorithm coupling a direct method (Newton's algorithm) with initial values, which would be isolated by Arnoldi's procedure. Then, it could be possible to compare the CPU times obtained from this method with those obtained from the author's procedure.

Moreover, the calculation times are still significant, and improvements have to be carried out in that way. As the computations are carried out in two steps, the indicator phase and the Newton method, future works will concern these two points. Thus, the computation of the indicator can be realized by using, for example, a linear solver well-adapted to repeated right-hand side problems [19] or with a reduced order model (POD [29], APR [32,33]). For the second point, some high order iterative algorithms $[34,35]$ can be used instead of the Newton method. These methods, based on homotopy and Padé approximants, have the advantage of converging to the solutions with lower CPU times than a classical iterative method such as Newton's method.

\section{REFERENCES}

1. Brezillon A, Girault G, Cadou JM. A numerical algorithm coupling a bifurcating indicator and a direct method for the computation of Hopf bifurcation points in fluid mechanics. Computers \& Fluids 2010; 39(7):1226-1240.

2. Cadou JM, Potier-Ferry M, Cochelin B. A numerical method for the computation of bifurcation points in fluid mechanics. European Journal of Mechanics-B/Fluids 2006; 25:234-254.

3. Jepson AD. Numerical Hopf bifurcation. Thesis, California Institute of Technology, 1981. 
4. Boppana VBL, Gajjar JSB. Global flow instability in a lid-driven cavity. International Journal for Numerical Methods in Fluids 2010; 62(8):827-853.

5. Fortin A, Jardak M, Gervais JJ, Pierre R. Localization of Hopf bifurcations in fluid flow problems. International Journal for Numerical Methods in Fluids 1997; 24:1185-1210.

6. Allery C, Cadou JM, Hamdouni A, Razafindralandy D. Application of the asymptotic numerical method to the Coanda effect study. Revue Européenne des Eléments finis 2004; 13:57-77.

7. Jackson CP. A finite-element study of the onset of vortex shedding in flow past variously shaped bodies. Journal of Fluid Mechanics 1987; 182:23-45.

8. Cochelin B. A path-following technique via an asymptotic-numerical method. Computer \& Structures 1994; 53(5):1181-1192.

9. Baker GA, Graves-Morris P. Padé approximants: Volume 59 of encyclopaedia of mathematics and its applications, 2nd ed. Cambridge University Press: Cambridge, 1996.

10. Elhage-Hussein A, Potier-Ferry M, Damil N. A numerical continuation method based on Padé approximants. International Journal of Solids and Structures 2000; 37:6981-7001.

11. Griewank A, Reddien G. The calculation of Hopf points by a direct method. IMA Journal of Numerical Analysis 1983; 3:295-303.

12. Bensaadi MEH. Méthode Asymptotique-Numérique pour le calcul de bifurcations de Hopf et de solutions périodiques. Thesis, Université de Metz, France, 1995.

13. Tri A. Méthodes Asymptotiques-Numériques pour les fluides visqueux incompressibles et la détection de la bifurcation de Hopf. Thesis, Université de Metz, France, 1996.

14. Cadou JM, Potier-Ferry M, Cochelin B, Damil N. ANM for stationary Navier-Stokes equations and with Petrov-Galerkin formulation. International Journal of Numerical Methods in Engineering 2001; 50:825-845.

15. Cadou JM, Duigou L, Damil N, Potier-Ferry M. Convergence acceleration of iterative algorithms. Application to thin shell analysis and Navier-Stokes equations. Computational Mechanics 2009; 43(2):253-264.

16. Potier-Ferry M, Damil N, Braikat B, Descamps J, Cadou JM, Cao HL, Hussein AE. Treatment of strong nonlinearities by the asymptotic numerical method. Comptes Rendus de l'Academie des Sciences Series IIB Mechanics Physics Chemistry Astronomy 1997; 324(3):171-177.

17. Najah A, Cochelin B, Damil N, Potier-Ferry MM. A critical review of asymptotic numerical methods. Archives of Computational Methods in Engineering 1998; 5:31-50.

18. Golub GH, Van Loan CF. Matrix Computations, 3rd ed. The Johns Hopkins University Press: Baltimore, MD, 1996.

19. Cadou JM, Potier-Ferry M. A solver combining reduced basis and convergence acceleration with applications to nonlinear elasticity. International Journal for Numerical Methods in Biomedical Engineering 2010; 26(12):1604-1617.

20. Damil N, Cadou JM, Potier-Ferry M. Mathematical and numerical connections between polynomial extrapolation and Padé approximants: applications in structural mechanics. Communications in Numerical Methods in Engineering 2004; 20:699-707.

21. Press WH, Teukolsky SA, Vetterling WT, Flannery BP. Numerical Recipes in Fortran 77: The Art of Scientific Computing, 2nd edn. Cambridge University Press: Cambridge, 1992. Chapter 9, 368-372.

22. Abouhamza A, Pierre R. A neutral stability curve for incompressible flows in a rectangular driven cavity. Math. Comp. Model 2003; 38:141-157.

23. Tiesinga G, Wubs FW, Veldman AEP. Bifurcation analysis of incompressible flow in a driven cavity by the Newton-Picard method. Journal of Computational and Applied Mathematics 2002; 140:751-772.

24. Nayar N, Ortega JM. Computation of selected eigenvalues of generalized eigenvalue problems. Journal of computational physics 1993; 108(1):8-14.

25. Lehoucq R, Sorensen DC, Yang C. Arpack User's Guide: Solution of large-scale eigenvalue problems with implicitly restarted Arnoldi methods. SIAM, Philadelphia, 1998.

26. Poliashenko M, Aidun CK. A direct method for computation of simple bifurcations. Journal of Computational Physics 1995; 121:246-260.

27. Bruneau CH, Saad M. The 2D lid-driven cavity problem revisited. Computers \&, Fluids 2006; 35:326-348.

28. Peng YF, Shiau YH, Hwang RR. Transition in 2-D lid-driven cavity flow. Computers \& Fluids 2003; 32:337-352.

29. Gervais JJ, Lemelin D, Pierre R. Some experiments with stability analysis of discrete incompressible flows in the lid-driven cavity. International Journal for Numerical Methods in Fluids 1997; 24:477-492.

30. Cazemier W, Verstappen RWCP, Veldman AEP. Proper orthogonal decomposition and low-dimensional models for driven cavity flows. Physics of Fluids 1998; 10(7):1685-1699.

31. Auteri F, Parolini N, Quartapelle L. Numerical investigation on the stability of singular driven cavity flow. Journal of Computational Physics 2002; 183:1-25.

32. Ryckelynck D. A priori hyperreduction method: an adaptive approach. Journal of Computational Physics 2005; 202(1):346-366.

33. Verdon N, Allery C, Béghein C, Hamdouni A, Ryckelynck D. Reduced-order modelling for solving linear and non-linear equations. International Journal for Numerical Methods in Biomedical Engineering; 27(1):43-58.

34. Mallil E, Lahmam H, Damil N, Potier-Ferry M. An iterative process based on homotopy and perturbation techniques. Computer Methods in Applied Mechanics and Engineering 2000; 190(13-14):1845-1858.

35. Cadou J-M, Damil N, Potier-Ferry M, Braikat B. Projection techniques to improve high-order iterative correctors. Finite Element in Analysis and Design 2004; 41(3):285-309. 